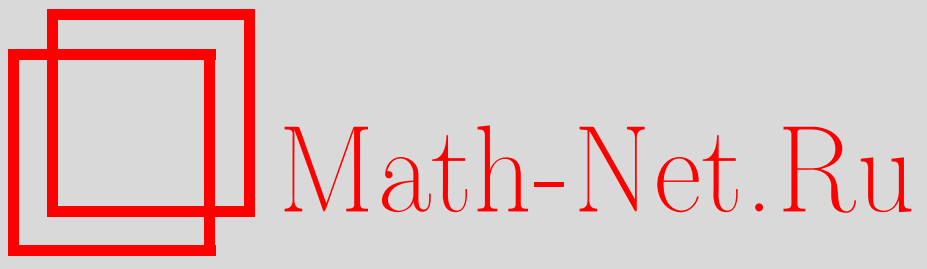

В. М. Бухштабер, Э. Г. Рис, Конструктивное доказательство обобщенного изоморфизма Гельфанда, Функи. анализ и его прил., 2001, том 35, выпуск 4, 20-25

DOI: https://doi.org/10.4213/faa269

Использование Общероссийского математического портала MathNet.Ru подразумевает, что вы прочитали и согласны с пользовательским соглашением

http://www.mathnet.ru/rus/agreement

Параметры загрузки:

IP : 52.90 .164 .192

26 апреля 2023 г., 12:56:09

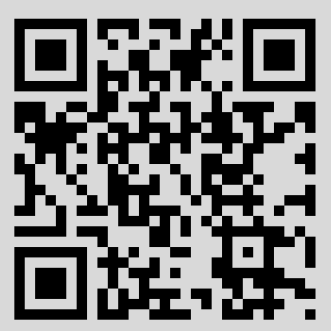


УДК 517.5

\title{
Конструктивное доказательство обобщенного изоморфизма Гельфанда
}

\author{
(C) 2001. В. М. Бухштабер, Э. Г. Рис
}

Пусть $X-$ компактное хаусдорфово пространство. Обозначим через $\mathbb{C}(X)$ алгебру непрерывных комплекснозначных функций на $X$ с sup-нормой.

В [1] в качестве следствия более общего результата мы получили, что образ вычисляющего отображения

$$
\mathscr{E}: \operatorname{Sym}^{n}(X) \rightarrow \operatorname{Hom}(\mathbb{C}(X), \mathbb{C})
$$

представляет собой множество непрерывных линейных отображений, которые являются фробениусовыми $n$-гомоморфизмами. В случае $n=1$ это очень хорошо известная теорема, принадлежащая И. М. Гельфанду. Естественно возникает вопрос, как для данного непрерывного фробениусова $n$-гомоморфизма $f: \mathbb{C}(X) \rightarrow \mathbb{C}$ построить такое множество $\left[x_{1}, \ldots, x_{n}\right]$, что $\mathscr{E}\left[x_{1}, \ldots, x_{n}\right]=f$. Цель настоящей статьи - ответить на этот вопрос и, таким образом, дать прямое доказательство теоремы (3.7) из [1].

1. Фробениусовы преобразования. Напомним некоторые основные определения из [1]. В тех случаях, когда встречаются утверждения, доказательства которых не приводятся здесь, мы отсылаем читателя к этой работе.

Пусть $f: A \rightarrow \mathbb{C}-$ линейное отображение, где $A-$ некоторая коммутативная алгебра. Определим индукцией по $n \in \mathbb{N}$ линейные отображения $\Phi_{n}(f): A^{\otimes n} \rightarrow$ $\mathbb{C}$, положив $\Phi_{1}(f)=f, \Phi_{2}(f)\left(a_{1}, a_{2}\right)=f\left(a_{1}\right) f\left(a_{2}\right)-f\left(a_{1} a_{2}\right)$ и для $n \geqslant 2$

$$
\begin{aligned}
\Phi_{n+1}(f) & \left(a_{1}, a_{2}, \ldots, a_{n+1}\right) \\
= & f\left(a_{1}\right) \Phi_{n}(f)\left(a_{2}, a_{3}, \ldots, a_{n+1}\right)-\Phi_{n}(f)\left(a_{1} a_{2}, \ldots, a_{n+1}\right) \\
& \quad-\Phi_{n}(f)\left(a_{2}, a_{1} a_{3}, \ldots, a_{n+1}\right)-\cdots-\Phi_{n}(f)\left(a_{2}, a_{3}, \ldots, a_{1} a_{n+1}\right) .
\end{aligned}
$$

ЗАМЕЧАНИЕ. Непосредственно из индуктивного определения следует, что если $f$ удовлетворяет условию $\Phi_{n}(f) \equiv 0$, то $\Phi_{n+1}(f) \equiv 0$.

ПреДЛОЖенИЕ 1.1. Если $f: A \rightarrow \mathbb{C}$ удовлетворяет условию $\Phi_{n+1}(f) \equiv 0$, то $f(1) \in\{0,1, \ldots, n\}$.

ОПРЕДЕЛЕНИЕ 1.2. Линейное отображение $f: A \rightarrow \mathbb{C}$ называется фробениусовым $n$-гомоморфизмом, если $\Phi_{n+1}(f) \equiv 0$ и $f(1)=n$.

ПреДЛОЖенИЕ 1.3. Пусть $f: A \rightarrow \mathbb{C}$ удовлетворяет условиям $\Phi_{n+1}(f) \equiv 0 u$ $f(1)=k ;$ тогда $\Phi_{k+1}(f) \equiv 0$.

ПреДЛОЖенИЕ 1.4. Если $f: A \rightarrow \mathbb{C}$ является суммой $n$ кольцевых гомоморфизмов $f_{i}: A \rightarrow \mathbb{C}, 1 \leqslant i \leqslant n$, то $f-$ фробениусов $n$-гомоморфизм.

Для дальнейшего нам нужно знать, как ведут себя фробениусовы $n$-гомоморфизмы на идемпотентных элементах алгебры $A$.

ПредЛожение 1.5. Пусть $f: A \rightarrow \mathbb{C}-$ фробениусов $n$-гомоморфизм. Тогда 
1) $f(e) \in\{0,1, \ldots, n\}$, где $e \in A-$ произвольный идемпотент;

2) $f\left(e_{1}\right)+\cdots+f\left(e_{r}\right) \leqslant n$, где $\left\{e_{1}, \ldots, e_{r}\right\}-$ произвольное множество ортогональных идемпотентов;

3) если $A=\mathbb{C}(X)$ и $X$ - конечное множество, то $f$ является суммой $n$ кольцеевых гомоморфизмов.

ДоКАЗАТЕЛЬСтво. Пусть $e \in A-$ идемпотент, т. е. $e^{2}=e$. Тогда

$$
\Phi_{n+1}(f)(e, \ldots, e)=(f(e)-n) \Phi_{n}(f)(e, \ldots, e)
$$

и по индукции $\Phi_{n+1}(f)(e, \ldots, e)=[f(e)]_{n}$, где $[x]_{n}-$ убывающий факториал $x(x-1) \cdots(x-n+1)$. Следовательно, если $\Phi_{n+1}(f) \equiv 0$, то $f(e)=k$ для некоторого целого $k$, такого, что $0 \leqslant k \leqslant n$.

Так как отображение $f$ линейно и сумма ортогональных идемпотентов также является идемпотентом, то свойство 2) вытекает из уже доказанного свойства 1).

Пусть $e_{x} \in \mathbb{C}(X)$ - идемпотентная функция, такая, что $e_{x}(x)=1$ и $e_{x}(y)=0$, если $y \neq x$. Тогда $f\left(e_{x}\right)=k_{x}$ для некоторого неотрицательного целого числа $k_{x}$ в силу свойства 1$)$; так как $1=\sum e_{x}$, мы имеем $\sum k_{x}=n$. Отображение $\widetilde{e}_{x}: \mathbb{C}(X) \rightarrow \mathbb{C}$, двойственное к $e_{x}$ (относительно этого базиса), является кольцевым гомоморфизмом, и $f=\sum k_{x} \widetilde{e}_{x}$. Таким образом, $f$ является суммой $n$ кольцевых гомоморфизмов.

Нам также потребуются следующие более специальные результаты:

ЛЕмма 1.6. Пусть $a_{1}, a_{2}, \ldots$ - некоторая последовательность элементов из $A$, такая, ито $a_{r+1} a_{r}=a_{r+1}$ для всех $r, u f: A \rightarrow \mathbb{C}-$ некоторое линейное отображение. Тогда

$$
\begin{aligned}
\Phi_{n+1}(f)\left(a_{k}, a_{k+1}, \ldots, a_{k+n}\right) & \\
& =\left(f\left(a_{k}\right)-n\right)\left(f\left(a_{k+1}\right)-n+1\right) \cdots\left(f\left(a_{k+n-1}\right)-1\right) f\left(a_{k+n}\right),
\end{aligned}
$$

u если $b a_{r}=0$ для всех $r$, mо

$$
\begin{aligned}
\Phi_{n+2}(f)\left(b, a_{k},\right. & \left.a_{k+1}, a_{k+2}, \ldots, a_{k+n}\right) \\
& =f(b)\left(f\left(a_{k}\right)-n\right)\left(f\left(a_{k+1}\right)-n+1\right) \ldots\left(f\left(a_{k+n-1}\right)-1\right) f\left(a_{k+n}\right) .
\end{aligned}
$$

ДокАЗАТЕЛЬство. Проведем прямые вычисления. Имеем

$$
\begin{aligned}
& \Phi_{n+1}(f)\left(a_{k}, a_{k+1}, \ldots, a_{k+n}\right)=f\left(a_{k}\right) \Phi_{n}(f)\left(a_{k+1}, \ldots, a_{k+n}\right) \\
&-\Phi_{n}(f)\left(a_{k} a_{k+1}, \ldots, a_{k+n}\right)-\cdots-\Phi_{n}(f)\left(a_{k+1}, \ldots, a_{k} a_{k+n}\right) .
\end{aligned}
$$

Так как $a_{r} a_{s}=a_{s}$ для $r<s$, то правая часть этого равенства равна

$$
\left(f\left(a_{k}\right)-n\right) \Phi_{n}(f)\left(a_{k+1}, \ldots, a_{k+n}\right)
$$

и по индукции мы получаем первое утверждение леммы. Второе утверждение следует из первого, если заметить, что

$$
\Phi_{n+2}(f)\left(b, a_{k}, a_{k+1}, \ldots, a_{k+n}\right)=f(b) \Phi_{n+1}(f)\left(a_{k}, a_{k+1}, \ldots, a_{k+n}\right) .
$$

Ограничение отображения $\Phi_{n}(f)$ на алгебру симметрических тензоров $\mathscr{S}^{n} A \subset$ $A^{\otimes n}$ обладает важным свойством мультипликативности, которое доказано в $[1]$ (теорема (2.8)). 
Теорема 1.7. Если $f: A \rightarrow \mathbb{C}$ является фробениусовым $n$-гомоморфизмом, то отображение

$$
\Phi_{n}(f) / n !: \mathscr{S}^{n} A \rightarrow \mathbb{C}
$$

является кольцевым гомоморфизмом.

2. Симметрические произведения компактных пространств. В этом разделе мы свяжем нашу теорию с изучением симметрических произведений.

Обозначим через $\Phi_{n}(A)$ множество всех фробениусовых $n$-гомоморфизмов из $A$ B $\mathbb{C}$.

Пусть, как и выше, $X$ - некоторое компактное хаусдорфово топологическое пространство, $\operatorname{Sym}^{n}(X)$ - симметрическое произведение $X^{n} / \Sigma_{n}$ и $\mathbb{C}(X)-$ алгебра непрерывных комплекснозначных функций на $X$ c sup-нормой. Тогда вычисляющее отображение

$$
\mathscr{E}: \operatorname{Sym}^{n}(X) \rightarrow \Phi_{n}(\mathbb{C}(X))
$$

определяемое формулой

$$
\left[x_{1}, \ldots, x_{n}\right] \rightarrow\left(g \rightarrow \sum g\left(x_{k}\right)\right),
$$

Является вложением. Ясно, что $\mathscr{E}$ функториально.

ТеОрема 2.1. Отображение

$$
\mathscr{E}: \operatorname{Sym}^{n}(X) \rightarrow \Phi_{n, c}(\mathbb{C}(X), \mathbb{C})
$$

является гомеоморфизмом, если в пространстве непрерывных линейных функционалов на $\mathbb{C}(X)$ рассматривается слабая топология.

СледствиЕ 2.2. В указанных выше условиях каждый непрерывный фробениусов $п$-гомоморфизм является суммой $n$ кольцевых гомоморфизмов.

ЗАмЕчАНИЕ. В случае $n=1$ это дает классический результат И. М. Гельфанда и $\mathscr{E}$ называется преобразованием Гельфанда. Тем не менее, по-видимому, «стандартные» доказательства того, что преобразование Гельфанда является гомеоморфизмом, нельзя приспособить к доказательству общего случая $n>1$. Действительно, большинство доказательств классического случая $n=1$ не «находит» точку $x \in X$, вычисление значений функций в которой задает кольцевой гомоморфизм $\mathbb{C}(X) \rightarrow \mathbb{C}$.

ДОКАЗАТЕЛЬСТвО ТЕОРЕмЫ 2.1. Выберем для компактного подмножества $K \subset X$ последовательность непрерывных функций $\varphi_{r}: X \rightarrow[0,1]$, такую, что

- $\varphi_{r}(K)=1$,

- $\varphi_{r}\left(\operatorname{supp}\left(\varphi_{r+1}\right)\right)=1$,

- $\bigcap_{r \geqslant 0} \operatorname{supp}\left(\varphi_{r}\right)=K$.

Мы называем такую последовательность огораживающей последовательностью для $K$. Очевидно, что $\varphi_{r} \varphi_{s}=\varphi_{s}$, если $r<s$.

Простое вычисление, использующее лемму 1.6, дает следующий результат:

ЛЕмма 2.3. Пусть $f: \mathbb{C}(X) \rightarrow \mathbb{C}$ - некоторый непрерывныцй фробениусов n-гомоморфизм, $\left\{\varphi_{r}\right\}$ - последовательность функицй, описанная выше, и $b_{r}=f\left(\varphi_{r}\right)$. Тогда если $r_{0}<r_{1}<\cdots<r_{n}$, то

$$
\left(b_{r_{0}}-n\right)\left(b_{r_{1}}-(n-1)\right) \cdots\left(b_{r_{n-1}}-1\right) b_{r_{n}}=0 .
$$


ПрЕДЛОЖЕНИЕ 2.4. Пусть $\left\{b_{r}\right\}$ - последовательность комплексных чисел, такая, что

$$
b_{0}=n, \quad\left(b_{r_{0}}-n\right)\left(b_{r_{1}}-(n-1)\right) \cdots\left(b_{r_{n-1}}-1\right) b_{r_{n}}=0
$$

для любых $r_{i}$, таких, что $r_{0}<r_{1}<\cdots<r_{n}$. Тогда существуют изелье числа $k$ u $N$, такие, что $b_{r}=k$ для всех $r \geqslant N$, причем $0 \leqslant k \leqslant n$.

ДОКАЗАТЕЛЬСТВО. ИспоЛЬЗУем индукцию по $n$.

При $n=1$ мы имеем $\left(b_{r}-1\right) b_{r+1}=0$ для всех $r$. Таким образом, либо $b_{r}=1$ для всех $r$, либо существует такое $r_{0}$, что $b_{r_{0}} \neq 1$. Но в этом случае, согласно приведенному выше тождеству, $b_{r_{0}+1}=0$, и тогда, снова используя это тождество, мы получаем, что $b_{r}=0$ для всех $r>r_{0}$, а это и требовалось.

В общем случае предположим, что существует такое $r_{0}$, что $b_{r_{0}} \neq n$. Тогда, согласно тождеству, $\left(b_{r_{1}}-(n-1)\right) \cdots\left(b_{r_{n-1}}-1\right) b_{r_{n}}=0$ для любых $r_{1}, \ldots, r_{n}$, таких, что $r_{0}<r_{1}<\cdots<r_{n}$. Следовательно, по индуктивному предположению существует такое $N$, что для всех $r>N$ мы имеем $b_{r}=k$ для некоторого целого $k$ в интервале $0 \leqslant k \leqslant n-1$.

Следовательно, огораживающая последовательность для $K$ определяет некоторое целое число $k$.

ЛЕмма 2.5. Для данного компактного подмножества $K \subset X$ цуелое число $k$ не зависит от выбора огораживающей последовательности $\varphi_{r}$.

ДокАЗАТЕЛьство. Рассмотрим две такие последовательности $\left\{\varphi_{r}\right\}$ и $\left\{\psi_{r}\right\}$. Выбирая соответствующие подпоследовательности, мы можем предположить, что $f\left(\varphi_{r}\right)=k$ и $f\left(\psi_{r}\right)=\ell$ для всех $r$ и что $\varphi_{r} \psi_{s}=\psi_{s}$ для $s \geqslant r$. Меняя местами, если необходимо, обозначения $\left\{\varphi_{r}\right\}$ и $\left\{\psi_{r}\right\}$, мы можем считать. что $k \leqslant \ell \leqslant n$. Тогда, поскольку $f$ является фробениусовым $n$-кольцевым гомоморфизмом, получаем

$$
\begin{aligned}
0 & =\Phi_{n+1}\left(\varphi_{1}, \varphi_{2}, \ldots, \varphi_{n-k}, \psi_{n-k}, \psi_{n-k+1}, \ldots, \psi_{n}\right) \\
& =(k-n)(k-(n-1)) \cdots(k-(k+1))(\ell-k)(\ell-(k-1)) \cdots(\ell-1) \ell .
\end{aligned}
$$

Из условия $k \leqslant \ell \leqslant n$ вытекает теперь, что $k=\ell$.

Комбинируя предыдущие результаты, мы можем определить вес $w_{f}(K)$ компактного множества $K \subset X$ относительно $f$ как предельное значение $k$ для любой из таких последовательностей $f\left(\varphi_{n}\right)$. Таким образом,

$$
w_{f}(K)=\lim _{n \rightarrow \infty} f\left(\varphi_{n}\right) .
$$

ПрЕДЛОЖЕНИЕ 2.6. Пусть $K u L-$ компактные подмножества в $X$. Тогда

(a) $w_{f}(K \cup L)+w_{f}(K \cap L)=w_{f}(K)+w_{f}(L)$;

(b) ecли $L \subset K$, mo $w_{f}(L) \leqslant w_{f}(K)$;

(c) если $K \cap L=\varnothing$, mo $w_{f}(K)+w_{f}(L) \leqslant n$.

ДОКАЗАТЕЛЬСТвО. Пусть $\left\{\varphi_{n}\right\}$ и $\left\{\psi_{n}\right\}$ - огораживающие последовательности для $K$ и $L$, соответственно.

(а) $\max \left(\varphi_{n}, \psi_{n}\right)$ и $\min \left(\varphi_{n}, \psi_{n}\right)$ являются огораживающими последовательностями для $K \cup L$ и $K \cap L$ соответственно. Доказываемый результат теперь следует из того, что $\max \left(\varphi_{n}, \psi_{n}\right)+\min \left(\varphi_{n}, \psi_{n}\right)=\varphi_{n}+\psi_{n}$.

(b) Предположим, что $f\left(\varphi_{r}\right)=k$ для всех $r \geqslant N$. Выберем $M$, такое, что $\varphi_{N}\left(\operatorname{supp}\left(\psi_{M}\right)\right)=1$, и рассмотрим последовательность $\left\{\varphi_{N}, \psi_{M}, \psi_{M+1}, \ldots\right\}$. 
Предел последовательности $f\left(\psi_{n}\right)$ есть $w_{f}(L)$, и так как $f\left(\psi_{n}\right)=w_{f}(K)$, то $w_{f}(L) \leqslant w_{f}(K)$.

(c) Это утверждение является прямым следствием двух предыдущих вместе с предложением 1.1 и того факта, что $w_{f}(X)=n$.

Наша конструкция позволяет сопоставить вес каждому одноточечному подмножеству в $X$. Обозначим через $S_{f}$ множество, состоящее из всех точек ненулевого веса, в которое каждая точка входит с кратностью, равной ее весу.

Лемма 2.7. Пусть $\psi: X \rightarrow \mathbb{C}-$ непрерьвная функция, пересечение носителя которой с $S_{f}$, является пустым множеством. Тогда $f(\psi)=0$.

ДокАЗАТЕЛЬСтво. Пусть $\left\{\varphi_{r}\right\}$ - последовательность функций, такая, что пересечение носителя каждой функции $\varphi_{r}$ с носителем функции $\psi$ пусто, $\varphi_{r}=1$ в окрестности множества $S_{f}$ и $\varphi_{r+1} \varphi_{r}=\varphi_{r+1}$ для каждого $r$. Следовательно, $\psi \varphi_{r}=0$ и, таким образом, $(1-\psi) \varphi_{r}=\varphi_{r}$. Однако существует $N$, такое, что $f\left(\varphi_{r}\right)=n$ для всех $r \geqslant N$. Используя определение фробениусова $n$-гомоморфизма и лемму 1.6, получаем, что

$$
0=f(\psi)\left(f\left(\varphi_{N}\right)-(n-1)\right)\left(f\left(\varphi_{N+1}\right)-(n-2)\right) \cdots f\left(\varphi_{N+n-1}\right) .
$$

Следовательно, $f(\psi)=0$.

ЛЕмма 2.8. Пусть $x_{0} \in S_{f} u \psi: X \rightarrow \mathbb{C}-$ непрерьвная функция, такая, umo $S_{f} \cap \operatorname{supp}(\psi)=\left\{x_{0}\right\}$. Tогда $f(\psi)=w_{f}\left(x_{0}\right) \psi\left(x_{0}\right)$.

ДокАЗАТЕЛЬСтвО. Пусть $\left\{\varphi_{r}\right\}$ - огораживающая последовательность для $\left\{x_{0}\right\}$. Рассмотрим функцию $\theta_{r}=\left(\psi-\psi\left(x_{0}\right)\right) \varphi_{r}$. Ее модуль не превосходит $\left|\psi-\psi\left(x_{0}\right)\right|$ и обращается в нуль вне некоторой окрестности точки $x_{0}$; ввиду непрерывности функции $\psi$ функции $\theta_{r}$ стремятся к 0 в sup-норме при $r \rightarrow \infty$. Следовательно, $f\left(\theta_{r}\right) \rightarrow 0$ при $r \rightarrow \infty$.

Но пересечение носителя функции $\psi\left(1-\varphi_{r}\right)$ с $S_{f}$ пусто; значит, согласно лемме 2.7, $f(\psi)=f\left(\psi \varphi_{r}\right)$. Используя теперь линейность функционала $f$, получаем $f(\psi)=f\left(\psi \varphi_{r}\right) f\left(\theta_{r}\right)+\psi\left(x_{0}\right) f\left(\varphi_{r}\right)$, и, таким образом, $f(\psi)=\psi\left(x_{0}\right) w_{f}\left(x_{0}\right)$.

СЛЕДСТВИЕ 2.9. Множество $S_{f}$ является $n$-точечным.

ДоКАЗАТЕЛЬСтво. Пусть $S_{f}=\left\{x_{1}, \ldots, x_{r}\right\}$ (без учета кратностей). Возьмем разбиение единицы $1=p_{1}+\cdots+p_{r}$, где пересечение носителя функции $p_{k}$ с $S_{f}$ равно $\left\{x_{k}\right\}$. Тогда если $f-$ фробениусов $n$-гомоморфизм, то в силу его линейности и леммы 2.8

$$
n=f(1)=f\left(p_{1}\right)+\cdots+f\left(p_{r}\right)=\sum_{k=1}^{r} w_{f}\left(x_{k}\right) p_{k}\left(x_{k}\right)=\sum_{k=1}^{r} w_{f}\left(x_{k}\right) .
$$

Следовательно, $S_{f}$ является $n$-точечным множеством.

Следующий результат завершает доказательство того, что отображение $\mathscr{E}$ из теоремы 2.1 является отображением на.

ПрЕДЛОЖЕНИЕ 2.10. Отображения $f u \mathscr{E}\left(S_{f}\right): \mathbb{C}(X) \rightarrow \mathbb{C}$ coвпадают.

ДоКАЗАТЕЛЬСтво. Каждая функция $\psi \in \mathbb{C}(X)$ может быть записана в виде суммы двух функций, носитель одной из которых близок к $S_{f}$, а другая обращается в нуль в окрестности множества $S_{f}$. Применяя две предыдущие леммы, мы получаем, что

$$
f(\psi)=\sum_{x \in S_{f}} w_{f}(x) \psi(x)
$$


и, тем самым, требуемый результат. Действительно, правая часть равенства может быть записана также как $\sum_{x \in X} w_{f}(x) \psi(x)$, поскольку $w_{f}(x)=0$ для $x \notin S_{f}$.

Благодарности. Настоящая статья опирается на исследования, которые были выполнены в ходе визитов первого автора в университет Эдинбурга, поддержанных Engineering and Physical Sciences Research Council и London Mathematical Society.

\section{ЛИТЕРАТУРА}

1. Buchstaber V. M., Rees E. G. The Gelfand map and symmetric products. Preprint, 2001; e-print uk.arXiv.org: math.CO/0109122.

Московский государственный университет, механико-математический факультет

Поступило в редакцию email: buchstab@mech.math.msu.su 10 сентября 2001 г.

Department of Mathematics and Mechanics,

Edinburgh University

email: elmer@maths.ed.ac.uk 